Wir müssen demnach annehmen, daß unsere parthenogenetischen Gastrulalarven, wohl durch Aufregulation, ebenfalls diploid sind. Damit unterscheiden sich die Kontrollen und die Versuchstiere lediglich in genetischer Hinsicht: Die Kontrollen haben je einen väterlichen und einen mütterlichen Gensatz, die parthogenetischen Tiere hingegen den doppelten mütterlichen Gensatz, in jedem Kern vereinigt.

Die Kernvariationsbreite bei den Seeigeln ist also keine einfache Funktion der Chromosomenzahl, sondern sie ist direkt von Erbfaktoren abhängig. Bedenken wir, daß die Gene als physiologisch wirksame Faktoren betrachtet werden können, so besteht zwischen einer identischen Verdoppelung des Gensatzes und einer verschiedenwertigen, wie sie der normale Befruchtungsvorgang liefert, ein Unterschied im Sinne einer physiologischen Mehrwertigkeit. Anderseits ist die Kerngröße eine Variable in bezug auf die physiologische Zellaktivität und schwankt in gewissen Grenzen je nach der Zelltätigkeit.

Fassen wir diesen Gedankengang kurz und formelartig zusammen, so können wir folgende Reihe aufstellen:

Kerngröße $=$ Funktion von physiologischer Leistung der Zelle = Funktion von Genbestand.

Die festgestellte Gesetzmäßigkeit, daß die Variationsbreiten der Kernradien bei isogener bzw. anisogener Diploidie sowie bei Haploidie und aniso-

\begin{tabular}{|c|c|c|c|c|c|}
\hline \multirow{2}{*}{$\mathrm{Nr}$. } & \multirow{2}{*}{$\begin{array}{c}\text { Zahl } \\
\text { der } \\
\text { Kerne }\end{array}$} & \multicolumn{2}{|c|}{ Kernradius } & \multirow{2}{*}{$\begin{array}{l}\text { Varia- } \\
\text { tions- } \\
\text { breite }\end{array}$} & \multirow{2}{*}{$\begin{array}{l}\text { Mittel- } \\
\text { wert } \\
\text { d. Kern- } \\
\text { größe }\end{array}$} \\
\hline & & kleinster & größter & & \\
\hline $\begin{array}{r}\text { I } \\
\text { II } \\
\text { III } \\
\text { IV } \\
\text { V }\end{array}$ & $\begin{array}{l}53 \\
55 \\
59 \\
56 \\
56\end{array}$ & $\begin{array}{l}5,3 \\
5,0 \\
5,7 \\
6,4 \\
6,3\end{array}$ & $\begin{array}{l}14,1 \\
14,2 \\
12,4 \\
12,8 \\
14,0\end{array}$ & $\begin{array}{l}8,8 \\
9,2 \\
6,7 \\
6,4 \\
7,7\end{array}$ & $\begin{array}{r}9,1 \\
9,3 \\
8,8 \\
9,4 \\
10,1\end{array}$ \\
\hline $\mathrm{a}$ & \multicolumn{2}{|c|}{ Mittelwert: } & \multicolumn{2}{|c|}{$\begin{array}{ll}\text { Kerngröße } & 9,34 \\
\text { Variationsbreite: } & 7,76\end{array}$} & \\
\hline $\begin{array}{r}\text { I } \\
\text { II } \\
\text { III } \\
\text { IV } \\
\text { V }\end{array}$ & $\begin{array}{l}56 \\
56 \\
57 \\
60 \\
57\end{array}$ & $\begin{array}{l}7,4 \\
8,1 \\
7,4 \\
8,6 \\
8,9\end{array}$ & $\begin{array}{l}12,2 \\
12,4 \\
10,4 \\
12,5 \\
11,5\end{array}$ & $\begin{array}{l}4,8 \\
4,3 \\
3,0 \\
3,9 \\
2,6\end{array}$ & $\begin{array}{r}9,3 \\
9,6 \\
8,8 \\
9,8 \\
10,2\end{array}$ \\
\hline $\mathrm{b}$ & \multicolumn{2}{|c|}{ Mittelwert: } & $\begin{array}{l}\text { rngröße: } \\
\text { riationsb. }\end{array}$ & te: $\begin{array}{r}9,60 \\
\text {, }\end{array}$ & \\
\hline
\end{tabular}

Tab. 1. Kernradien und Variationsbreite bei normal befruchteten (a) und parthenogenetischen (b) Eiern.

gener Diploidie sich wie 1:2 verhalten, kann z. Z. nur für Seeigel angenommen werden, eine Verallgemeinerung des Befundes setzt weitere Versuche bei anderen systematischen Gruppen voraus.

\title{
Über die Autoxydation ungesättigter Bausteine des menschlichen Gehirns
}

\begin{abstract}
Von Rolf Schulz und Günther Wilke*
Aus dem Organisch-Chemischen Institut der Universität Mainz (Direktor: Prof. Dr. W. K e r n) und dem Max-Planck-Institut für Hirnforschung, Gießen, Physikalisch-Chemisches Laboratorium (Prof. Dr. G. W il k e)

(Z. Naturforschg. 10 b, 427-429 [1955]; eingegangen am 2. März 1955)

Die geschilderten Versuchsergebnisse zeigen, daß die aus Gehirnphosphatiden isolierten hochungesättigten Fettsäuren unter den Bedingungen der Autoxydation polymerisieren. Auch die natürlich vorkommenden Glycerinphosphatide erleiden eine Molekülvergrößerung, die mit einer Viskositätserhöhung verbunden ist. Die Versuche demonstrieren die grundsätzliche Möglichkeit, daß ungesättigte Bausteine des menschlichen Gehirns durch Autoxpolymerisation in einen viskosen, quellbaren Zustand übergeführt werden. Ob die Polymerisations-Fähigkeit der ungesättigten Fettsäuren des Gehirns mit den Vorgängen bei der Hirnschwellung in Zusammenhang gebracht werden kann, wird weiter untersucht.
\end{abstract}

D ie Autoxydation ungesättigter Fettsäuren und ihrer Derivate (Ester und Glyceride) ist wegen ihrer theoretischen und praktischen Bedeutung schon von verschiedenen Autoren bearbeitet worden ${ }^{1}$. In der ersten Reaktionsstufe liegt nach dem heutigen Stand der Erkenntnis zweifellos eine Radikal-Kettenreaktion vor. Die Folgereaktionen der primär geFußnote ${ }^{*}$ und 1 auf Seite 428 
bildeten Peroxyde führen bei bestimmten Verbindungen unter Verknüpfung der Moleküle zu hochmolekularen Produkten und damit zu Gelbildung, Filmbildung oder Verharzung. Treibs ${ }^{2}$ spricht hier von Autoxpolymerisation. Über den Mechanismus dieses Vorganges sind noch keine endgültigen Aussagen möglich.

Die „Hirnschwellung“ - eine lebensbedrohliche Reaktionsform der Hirnmaterie auf verschiedenartige Schädlichkeiten - ist pathogenetisch noch weitge-

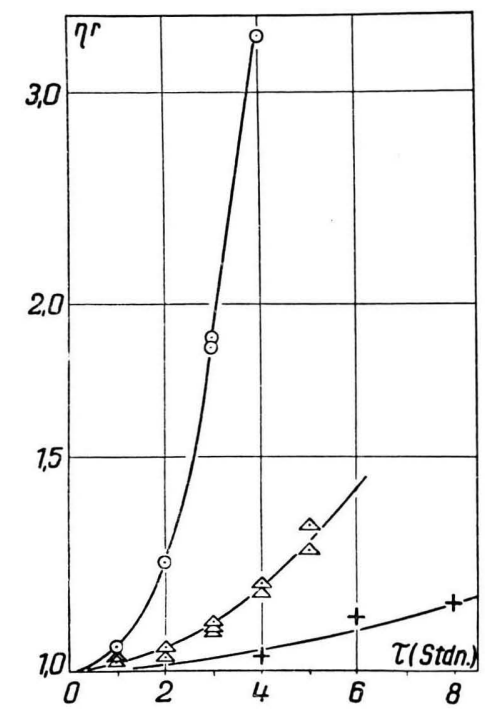

Abb. 1. Viskosität hochungesättigter Fettsäuren in Abhängigkeit von der Autoxydationsdauer bei verschiedenen Temperaturen. Jeweils $1,5 \mathrm{ccm}$ Fettsäure mit $\mathrm{O}_{2}$ durchströmt; + : bei $26^{\circ} \mathrm{C} ; \triangle$ : bei $36^{\circ} \mathrm{C}$; $\bigcirc$ : bei $46^{\circ} \mathrm{C}$. Viskosität bei Zimmertemperatur gemessen.

hend ungeklärt. Wir vermuten, daß dieser Vorgang mit der Bildung hochmolekularer, mehr oder weniger vernetzter Substanzen einhergeht ${ }^{3}$. Im Zusammenhang mit dieser Hypothese haben wir das Verhalten im Gehirn vorkommender ungesättigter Verbindungen unter den Bedingungen der Autoxydation untersucht. Für diese Untersuchung kamen vor allem die von $\mathrm{K}$ le $\mathrm{n} \mathrm{k}^{4}$ und Mitarbb. in den Glycerinphospha-

* Für die Hilfe bei Durchführung der Versuche danken wir Frau G is e l a K l e m m. - Die Untersuchungen wurden durch die Deutsche Forschungsgemein$\mathrm{s} \mathrm{ch}$ a f t unterstützt.

1 W. K e r n u. H. W ill e r s i n n, Makromolekulare Chem. 15, 1, 15, 36 [1955]; dort weitere Literatur; W. Franke, Ergebnisse der Enzymforschung, Bd. 12, Leipzig 1951, S. $89 \mathrm{ff}$.

2 W. Treibs, Ber. dtsch. chem. Ges. 75, 331 [1942]; Fette u. Seifen 52, 549 [1950]; 54, 3 [1952]. tiden des Gehirns aufgefundenen $\mathrm{C}_{20^{-}}$und $\mathrm{C}_{22}$-Polyensäuren in Betracht, die bis zu 6 isolierte Doppelbindungen ${ }^{5}$ im Molekül enthalten. Wir prüften die freien hochungesättigten Fettsäuren, das noch nicht aufgetrennte Gemisch der Methylester aller in den Phosphatiden vorkommenden Fettsäuren und schließlich die Glycerinphosphatide selbst. Die Substanzen wurden im Laboratorium von Prof. K le n k nach der von ihm ausgearbeiteten Methode aus menschlichem Hirnmaterial isoliert.

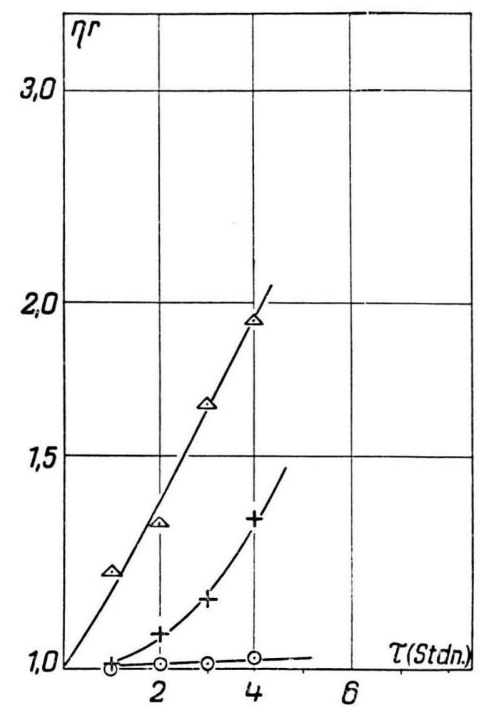

Abb. 2. Viskosität hochungesättigter Fettsäuren in Abhängigkeit von der Autoxydationsdauer in Gegenwart von Katalysator und Inhibitor. - Jeweils $1,3 \mathrm{ccm}$ eines Gemisches von Fettsäuren und Phthalsäuredimethylester (1:1) bei $46^{\circ} \mathrm{C}$ mit $\mathrm{O}_{2}$ durchströmt; + : ohne Zusatz; $\triangle$ : mit $50 \mathrm{mg}$ Benzoylperoxyd; $\bigcirc$ : mit $50 \mathrm{mg}$ Hydrochinon.

\section{Methodik}

1-2 g der zu untersuchenden Substanzen wurden, teils unter Zusatz eines Lösungsmittels, in Saugröhrchen gefüllt und bei $46^{\circ}, 36^{\circ}$ und $26^{\circ} \mathrm{C}$ mittels einer Kapillare Sauerstoff eingeleitet. Nach bestimmten Zeiten wurde mit einem modifizierten Kapillar-Viskosimeter im Reaktionsgefäß die Viskosität gemessen. Diese wird als relative Viskosität

$$
\eta_{\mathrm{r} \tau}=\frac{t_{\tau}}{t_{0}}
$$

3 G. W il k e u. H. G e n s e l, Naturwissenschaften 38, 532 [1951]; Arch. Psychiatr. u. Z. Neur. 187, 424 [1952]; G. W il k e, Dtsch. Z. Nervenheilkunde 168, 459 [1952].

${ }^{4}$ E. K l e n k, Hoppe-Seyler's Z. physiol. Chem. 200, 51 [1931]; 206, 25 [1932].

5 E. K lenk u. W. B on gard, Hoppe-Seyler's Z. physiol. Chem. 291, 104 [1952]; E. K l e n k, Naturwissenschaften 41, 68 [1954]; E. K lenk u. F. Lind la r, Hoppe-Seyler's Z. physiol. Chem. 299, 74 [1955]. 
angegeben, wobei $t_{\tau}$ die Durchlaufzeit (in sec) nach der Reaktionszeit $\tau$ (in Stdn.) ist und $t_{0}$ die Durchlaufzeit zu Versuchsbeginn. Zur Zeit $\tau=0$ ist $\eta_{\mathrm{r}}=1 ; \eta_{\mathrm{r}}$ wächst mit steigender Viskosität.

\section{Ergebnisse}

Die freien hochungesättigten Fettsäuren (Jodzahl 257) stellen gelbliche Öle dar. Bei der Behandlung mit Sauerstoff werden sie in zunehmendem Maße viskos und erstarren schließlich zu Gelen. Diese quellen stark auf, wenn man sie in Wasser legt. Die Viskositätszunahme in Abhängigkeit von der Zeit bei verschiedenen Reaktionstemperaturen ist in Abb. 1 dargestellt. Schon bei geringer Viskositätserhöhung lassen sich in dem Reaktionsgemisch Peroxyde nachweisen (mit Kaliumjodid in Eisessig). Die Menge der Peroxyde nimmt mit zunehmender Viskosität zu.

Die Viskositätszunahme kann durch Zusatz von Benzoylperoxyd beschleunigt und durch Hydrochinon gehemmt werden (Abb.2). Bei der Durchströmung mit Kohlensäure statt mit Sauerstoff bleibt die Viskosität auch in Gegenwart von Benzoylperoxyd unverändert.

Aus diesem Verhalten darf geschlossen werden, daß bei der Einwirkung von Sauerstoff auf die hochungesättigten Fettsäuren der Gehirnphosphatide eine Autoxydation erfolgt, die mit einer Polymerisation verknüpft ist. Es handelt sich also um eine Autoxpolymerisation im Sinne von Treibs ${ }^{2}$. Die Hemmbarkeit mit Hydrochinon und die Beschleunigung mit
Peroxyden deutet darauf hin, daß die Reaktion durch Radikale gestartet wird.

Die als Zwischenprodukte bei der Isolierung der hochungesättigten Fettsäuren anfallenden Gemische der gesättigten und ungesättigten Fettsäuren bzw. deren Ester wurden ebenfalls der Autoxydation unterworfen. Hier war es jedoch erforderlich, ein Lösungsmittel zuzusetzen. Als geeignet erwies sich Phthalsäuredimethylester. Es zeigte sich, daß unter obigen Bedingungen eine geringe, aber deutliche Viskositätserhöhung eintritt sowie eine Bildung von Peroxyden. Setzt man 5-10\% Benzoylperoxyd (bezogen auf das Substrat) zu und leitet Sauerstoff hindurch, so erfolgt ein schneller Viskositätsanstieg.

Die Autoxydation der Glycerinphosphatide wurde in Palmitinsäuremethylester als Lösungsmittel bei $36^{\circ}$ und $46^{\circ} \mathrm{C}$ durchgeführt. Nach $40 \mathrm{Stdn}$. ist mit der angewandten Methode noch keine Veränderung festzustellen. Man kann aber zeigen, daß bei Zugabe von Initiatoren der gleiche Vorgang wie bei den Fettsäuren ausgelöst wird. Setzt man beispielsweise 10\% Benzoylperoxyd hinzu, so steigt die Viskosität bei $46^{\circ} \mathrm{C}$ nach $8 \mathrm{Stdn}$. auf $\eta_{\mathrm{r}}=1,5$ und bei $36^{\circ} \mathrm{C}$ auf $\eta_{\mathrm{r}}=1,3$.

Die Versuche mit den Phosphatiden sind noch nicht abgeschlossen; sie werden mit einer anderen Methode, welche auch die Gesamtmenge des aufgenommenen Sauerstoffs zu messen gestattet, unter Verwendung physiologischer Katalysatoren fortgesetzt.

\title{
Über zwei Bedingungen der Digitonin-Hämolyse des einzelnen Erythrocyten
}

\section{Mitt.: Zur Analyse der Brooks-Ponderschen Hämolyseformel}

\author{
Von G. Ruhenstroth-Bauer und P. M. Breitenfeld \\ Aus dem Max-Planck-Institut für Biochemie, Tübingen \\ (Z. Naturforschg. 10 b, 429-432 [1955]; eingegangen am 7. Juli 1955)
}

\begin{abstract}
1. Wenige Min. nach Mischen einer Erythrocyten-Aufschwemmung mit einer Digitoninlösung ist das gesamte Digitonin durch die Oberfläche der Zellen gebunden. Es wird die Bedeutung dieser Tatsache für die Brooks-Pondersche Hämolyseformel besprochen.

2. Das $n$ der Brooks-Ponderschen Hämolyseformel ist außer von der Zell- und Hämolysinart auch von der Zellkonzentration abhängig. Damit ist eine 2. Größe gefunden, die bei der Einwirkung des Digitonins auf den Erythrocyten zellkonzentrations-abhängig ist.
\end{abstract}

S chmidt-Thomé ${ }^{1}$ hat 1942 angenommen, daß die Zahl der Cholesterinmolekeln eines Erythrocyten mit der Zahl der Digitoninmolekeln gut übereinstimmt, die von diesem maximal gebunden

1 J. S c h mid t - T h o m é, Hoppe-Seyler's Z. physiol. Chem. 275, 183 [1942]. werden können. Deshalb hielt er das Cholesterin der Erythrocyten-Membran für den primären und einzigen Angriffspunkt des Digitonins. In der folgenden Mitteilung ${ }^{2}$ wird gezeigt werden, daß jene UUberein-

\footnotetext{
2 P. M. Breitenfeld u. G. RuhenstrothB a u e r, Blut, im Druck.
} 